\title{
K-SHELL PHOTOIONIZATION OF Na-LIKE TO Cl-LIKE IONS OF Mg, Si, S, Ar, AND Ca
}

\author{
M. C. Witthoeft ${ }^{1}$, J. García ${ }^{1}$, T. R. Kallman ${ }^{1}$, M. A. Bautista ${ }^{2}$, C. Mendoza ${ }^{3}$, P. Palmeri ${ }^{4}$, and P. Quinet ${ }^{4}$ \\ ${ }^{1}$ NASA Goddard Space Flight Center, Code 662, Greenbelt, MD 20771, USA \\ 2 Department of Physics, Western Michigan University, Kalamazoo, MI 49008, USA \\ ${ }^{3}$ Centro de Física, Instituto Venezolano de Investigaciones Científicas (IVIC), Caracas 1020A, Venezuela \\ ${ }^{4}$ Astrophysique et Spectroscopie, Université de Mons-UMONS, B-7000 Mons, Belgium \\ Received 2010 September 15; accepted 2010 October 15; published 2010 December 22
}

\begin{abstract}
We present $R$-matrix calculations of photoabsorption and photoionization cross sections across the $\mathrm{K}$ edge of $\mathrm{Mg}$, $\mathrm{Si}, \mathrm{S}, \mathrm{Ar}$, and $\mathrm{Ca}$ ions with more than 10 electrons. The calculations include the effects of radiative and Auger damping by means of an optical potential. The wave functions are constructed from single-electron orbital bases obtained using a Thomas-Fermi-Dirac statistical model potential. Configuration interaction is considered among all states up to $n=3$. The damping processes affect the resonances converging to the K-thresholds causing them to display symmetric profiles of constant width that smear the otherwise sharp edge at the photoionization threshold. These data are important for the modeling of features found in photoionized plasmas.
\end{abstract}

Key words: atomic data - atomic processes - line: formation - X-rays: general

Online-only material: color figures, supplemental data (FITS) file (tar.gz)

\section{INTRODUCTION}

The current fleet of X-ray telescopes (Chandra, XMMNewton, Suzaku) has revealed complex spectra from astrophysical sources such as active galactic nuclei (AGNs) and X-ray binaries. Besides the ubiquitous $\mathrm{Fe}$ absorption features, many other elements from $\mathrm{C}$ to $\mathrm{Ni}$ have been observed in multiple ionization stages; see, for example, the 900 ks Chandra spectrum of NGC 3783 from Kaspi et al. (2002). In order to accurately model systems producing such spectra, atomic data are needed for all of these species, particularly the resonance structure leading to the absorption features. Due to the wavelengths of these features, the relevant atomic data involve the K-shell of species from $\mathrm{C}$ to $\mathrm{Ni}$ where the currently available data have been of limited accuracy. Over the last several years, our group has worked on providing these data. We have calculated energy levels, wavelengths, Einstein A-coefficients, Auger rates, and photoionization cross sections. Complete data sets have been computed for all ion stages of Fe (Bautista et al. 2003; Palmeri et al. 2003a, 2003b; Mendoza et al. 2004; Bautista et al. 2004; Kallman et al. 2004), O (García et al. 2005), and N (García et al. 2009). There has also been recent work on the $C$ sequence by Hasoglu et al. (2010). This work is an extension of Palmeri et al. (2008) and Witthoeft et al. (2009) where we present data for the Li-like to Ne-like ions of $\mathrm{Ne}, \mathrm{Mg}, \mathrm{Si}, \mathrm{S}, \mathrm{Ar}$, and $\mathrm{Ca}$. Here we continue these calculations for the 23 non-neutral, Na-like to $\mathrm{Cl}$-like ions of the same elements.

$\mathrm{K}$-shell features of the ion stages covered here have been detected in X-ray spectra of astronomical objects, most often due to fluorescent line emission. Notable examples include the detection of $\mathrm{K} \alpha$ emission from the ions $\mathrm{Si}$ II-V in the X-ray binary Vela X-1 (Paerels \& Kahn 2003). This reveals the existence of a wide range of ionization states coexisting in this system, and can best be explained if the emitting gas is highly clumped. $\mathrm{K} \alpha$ lines from $\mathrm{S}$ IV-VI, Ar VI-VIII, and Ca VI-VII have been detected in the X-ray binary 4U1700-37 (Boroson et al. 2003). The flux in these lines is correlated with the flux in the X-ray continuum, thus reinforcing the fluorescent origin. The $\mathrm{K}$ edge of neutral $\mathrm{Ne}$ has been detected from interstellar gas along the line of sight to bright X-ray binaries (Juett \& Chakrabarty 2003) and provides evidence for local enhancements in the interstellar $\mathrm{Ne} / \mathrm{O}$ elemental abundance. The data we present here are important both for its direct effect on observed spectra via absorption features, and also through its effect on ionization balance calculations, which in turn affect many of the observables from these elements. In particular, it is the detailed resonance and edge structure, missing in previous calculations, which is most important.

There have been a large number of calculations for these systems going back to the 1920 s, but they are confined to the non-resonant background and few cross the K-edge region. For the ions considered in this work, there is good agreement in the background cross section both above and below the K edge and are well characterized by the fits of Verner \& Yakovlev (1995). Measurements have been mainly confined to the neutral systems and for high photon energies. A comprehensive assessment and fitting of measurements has been performed by Veigele (1973).

There has been recent progress measuring the resonance structure near the $\mathrm{K}$ edge of Li-like systems: $\mathrm{C}^{3+}$ (Müller et al. 2009) and $B^{2+}$ (Müller et al. 2010), as well as Be-like $C^{2+}$ (Scully et al. 2005). $R$-matrix calculations performed as part of those works are in good agreement with the measurements apart from small discrepancies in some resonance positions and heights. With the advances of free electron lasers to produce $\mathrm{X}$-rays (Kanter et al. 2006), we expect it will be possible over the next several years to perform similar experiments for the species under investigation here.

Tables of the total and partial cross sections accompany this paper as online tables available as a supplemental data file. Full data sets are also available through the XSTAR atomic database (Bautista \& Kallman 2001). ${ }^{5}$

\section{NUMERICAL METHODS}

The $R$-matrix method employed here is the same as our previous K-shell calculations and described in detail in Bautista

\footnotetext{
5 http://heasarc.nasa.gov/lheasoft/xstar/xstar.html
} 


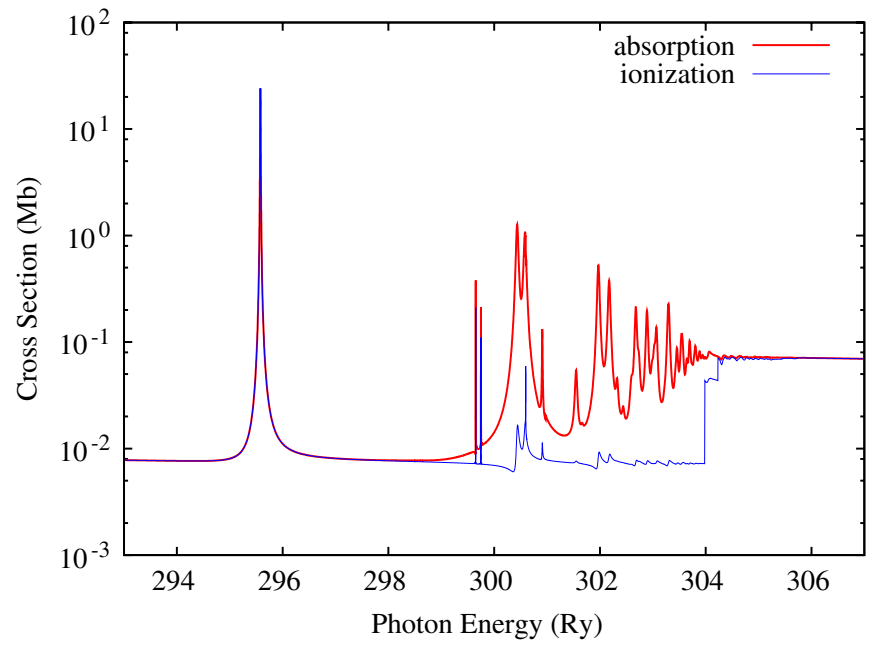

Figure 1. Photoabsorption (thick, red) and photoionization (thin, blue) cross sections of P-like Ca near the $\mathrm{K}$ edge.

(A color version of this figure is available in the online journal.)

et al. (2003). The $R$-matrix method is based on the closecoupling approximation of Burke \& Seaton (1971) which is solved numerically following Burke et al. (1971) and Berrington et al. (1974, 1978, 1987). Due to the complexity of these M-shell ions, the scattering calculations are carried out in $L S$-coupling except for the Na-like ions where we use the Breit-Pauli $R$-matrix framework (BPRM). The main limitation of the $L S$ calculations is that the results are not fine-structure resolved. As we will show in the following section, the total cross section is not sensitive to the configuration (or coupling scheme) of the initial state. However, for modeling purposes, we would like to have cross sections resolved to the fine-structure level, so we split the cross section according to Rau (1976). Our wave functions were obtained using AUTOSTRUCTURE (Badnell 1986, 1997) where the term (or level) energies have been adjusted to match NIST (Ralchenko et al. 2008) when possible. For energies which are not available in NIST, most importantly those for the K-hole states, we adjust to match the relativistic Hartree-Fock calculations of Palmeri et al. (2008).

As in our previous calculations, the effect of radiative and spectator Auger decay on resonances is included using the optical potential of Gorczyca \& Badnell (1996, 2000). Here, the resonance energy with respect to the threshold acquires an imaginary component which is the sum of the radiative and Auger widths of the core. The radiative widths are computed within the $R$-matrix calculations following Robicheaux et al. (1995). The Auger widths are provided from the relativistic Hartree-Fock calculations of Palmeri et al. (2008). These effects are referred to as radiative and Auger damping since they cause resonances in the photoionization cross section to become smaller in area. The damping also makes it necessary to calculate both the photoabsorption and photoionization cross sections since not every absorbed photon will result in a photoelectron.

We use the $R$-matrix computer package of Berrington et al. (1995) for the inner region calculation and the asymptotic region code STGBF0DAMP (Gorczyca \& Badnell 1996; N. R. Badnell 1996, unpublished) is used to compute the photoabsorption and photoionization cross sections including the effects of both radiation and Auger dampings. The target expansion for each ion includes the $1 s^{2} 2 l^{8} 3 l^{N}, 1 s^{2} 2 l^{7} 3 l^{N+1}$, and $1 s 2 l^{8} 3 l^{N+1}$ configurations, where $N+10$ is the number of electrons in the photoionized (target) ion. Only configurations with at most a single $3 d$ electron are included. The number of target states therefore can be as few as 43 levels (Na-like) or as many as 132 terms (P-like). We include enough continuum basis orbitals to span from threshold to at least three times the energy of the $\mathrm{K}$ edge. This choice gives reliable cross sections up to at least 1.5 times the energy of the $\mathrm{K}$ edge. Partial and total cross sections are calculated from all terms in the ground configuration of the parent ion.

At this point, we would like to make a note about the accuracy of the K-edge energy. For neutral systems where measurements exist, we can be fairly confident of the edge position. However, for ions, we must rely on calculated positions. Due to the magnitude of these energies, differences on the order of a percent yield uncertainties in the edge of a few Rydberg. In the present calculations, the energy of the edge is determined by two quantities: the ionization potential calculated by $R$-matrix and the energy of the first K-hole target state which we take from Palmeri et al. (2008). Since the ionization potential is known, we can (and do) adjust our cross sections by the difference with observations ( $\sim 0.2 \mathrm{Ry}$ ) when transferring our data to modeling codes. It is more difficult to judge the accuracy of the K-vacancy target state energies except to say that we expect the accuracy to improve as the charge state increases; see Palmeri et al. (2008) for an assessment of the accuracy. We estimate that the resonance and edge positions of the singly ionized systems could be shifted by up to 1 Ry from their true values (T. W. Gorczyca 2010, private communication). We consider this to be the limiting error for these systems. The accuracy of the edge and resonance positions should improve significantly for the higher charge states.

\section{RESULTS}

The cross sections reported here contain many of the same features shown in calculations from other ions and elements. Inclusion of radiation and Auger damping broadens resonances near the $\mathrm{K}$ edge and makes them more symmetric. The damping is also important in separating the absorption and ionization cross sections. In Figure 1, we show these cross sections for the ${ }^{4} S$ ground term of P-like $\mathrm{Ca}$. There is little difference between absorption and ionization for the first, $\mathrm{K} \beta$ resonance which indicates that damping plays little role here. For the near-edge resonances, however, the photoionized resonances are nearly damped away entirely while the strongly broadened absorption resonances lead to the characteristic smearing of the $\mathrm{K}$ edge. Small resonances can be observed above the edge in both absorption and ionization. These features are either typical resonances converging onto a higher-energy edge or resonances belonging to doubly excited states which are accessible via mixing. They are observed in all of the calculations, but rarely contribute much to the total cross section.

In Figure 2, we show the photoabsorption cross section for each of the Na-like ions. The behavior of the cross section across the iso-electronic sequence is quite regular. The background cross section above and below the K edge increases with nuclear charge and the width of the resonance region also increases with $Z$. However, the background cross section is known to be insensitive to the number of electrons in the valence shell (Missavage et al. 1977; Nasreen et al. 1989). If we were to plot the cross sections for all iso-electronic sequences in this same figure, it would look much the same. The exception is below the L edge for sequences with more than 12 electrons where the inner-shell $(3 s)$ electron has the same principal quantum number as the valence $(3 p)$ electron. In these cases, the insensitivity is lost (Pradhan et al. 2009). 


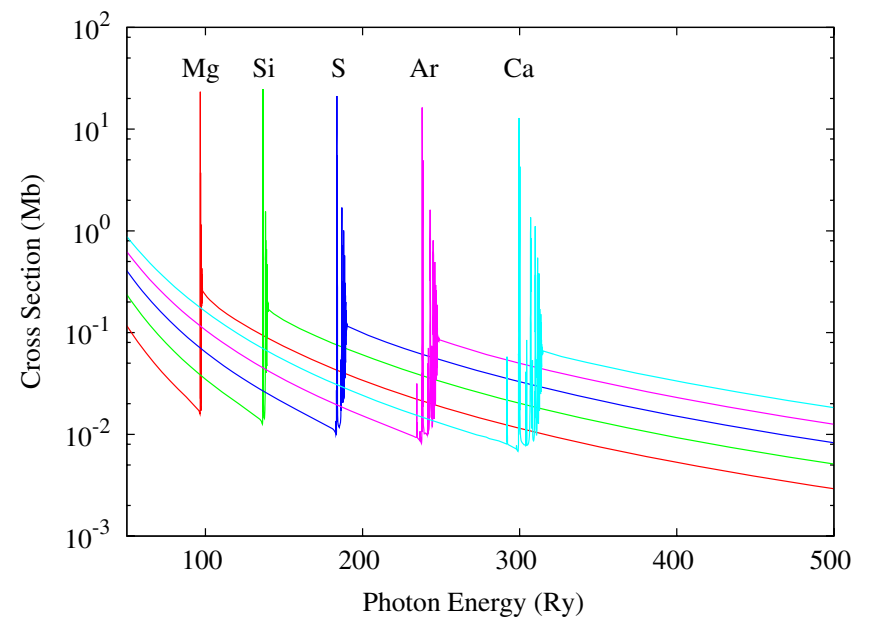

Figure 2. Photoabsorption cross sections for all Na-like ions included in this study.

(A color version of this figure is available in the online journal.)

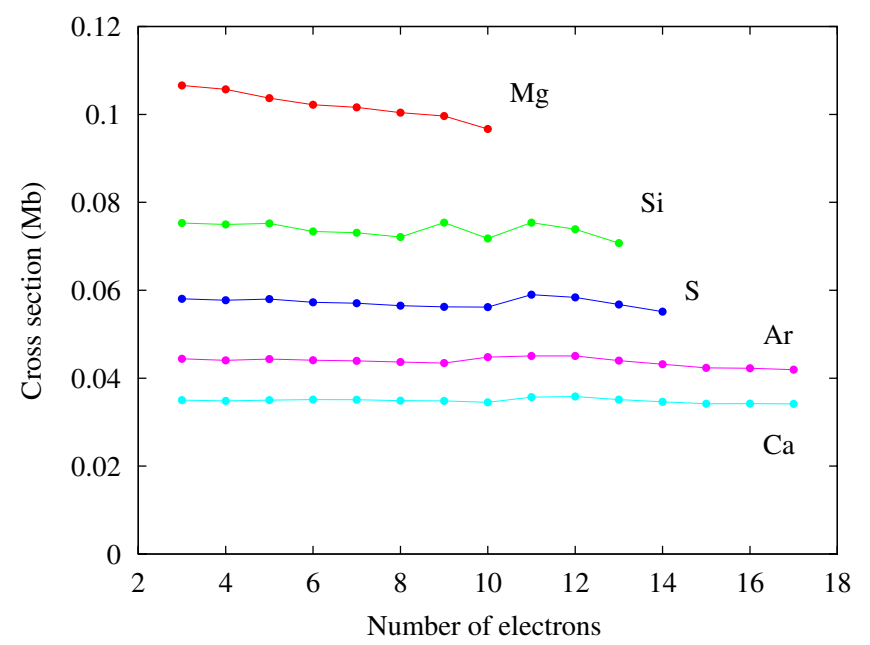

Figure 3. Background cross section at a fixed energy above the K edge across iso-nuclear sequences. The photon energies for each sequence are $128 \mathrm{Ry}(\mathrm{Mg})$, 178 Ry (Si), 233 Ry (S), 300 Ry (Ar), and 375 Ry (Ca).

(A color version of this figure is available in the online journal.)

In Figure 3, we show the background cross section at a fixed energy above the $\mathrm{K}$ edge where results from Witthoeft et al. (2009) are used for ions with 10 electrons or fewer. We find the background cross section for each sequence to be constant within $10 \%$. Note that the choice of energy is arbitrary and is different for each sequence so no conclusions should be drawn about the relative cross sections between sequences. Nor does the choice of energy affect the agreement, we find better than $10 \%$ agreement between the cross sections at all energies where we can make comparisons. Only the $\mathrm{Mg}$ sequence shows a steady downward trend of the cross section with an increasing number of electrons, but the total drop is only $10 \%$ so it is difficult to make any conclusions. The remaining sequences show no trend with the number of electrons. Note that we are starting with the Li-like ions; if we also showed the cross section for photoionization of the He-like ions, we would expect to see differences since there is no inner shell.

Previous experiments and calculations for K-shell photoionization were more concerned with high-energy behavior than the detailed structure of the cross section near the $\mathrm{K}$ edge. These results are generally in good agreement and have been charac-

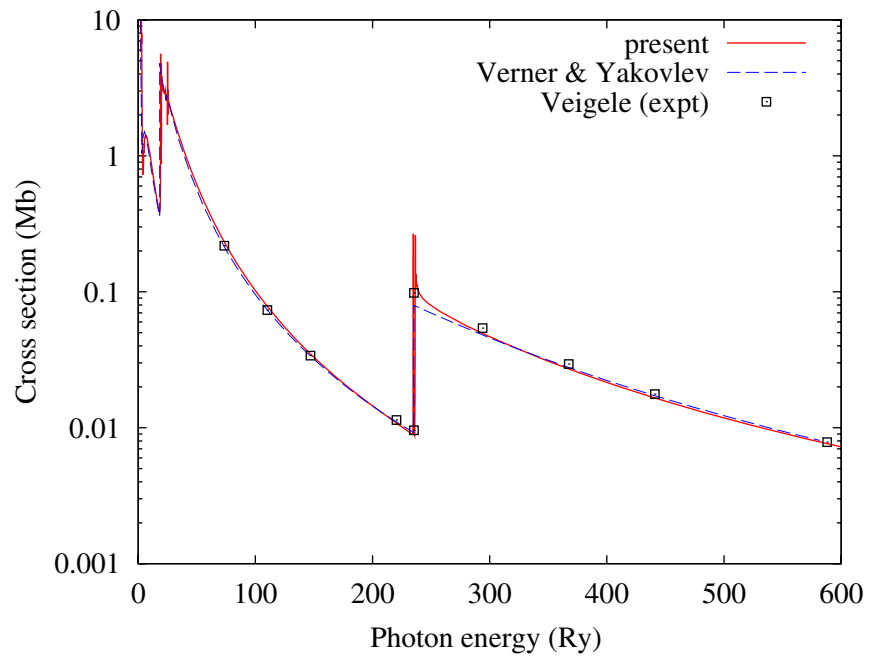

Figure 4. Photoabsorption cross section for Cl-like Ar. Solid (red) curve gives the present, $R$-matrix results, the dashed (blue) curve is from Verner \& Yakovlev (1995), and the boxes give the result from the experimental compilation of Veigele (1973).

(A color version of this figure is available in the online journal.)

terized by the fits of Verner \& Yakovlev (1995). We want to compare our results with experiment, but measurements have only been taken for neutral systems not calculated here. However, we can take advantage of the finding above and compare our singly ionized results with the neutral measurements as a test of the background. In Figure 4, we show our cross section for Cl-like Ar compared to the photoabsorption cross sections of neutral Ar given by Verner \& Yakovlev (1995) and the fit to experimental data by Veigele (1973). There is very good agreement between the present results and Verner \& Yakovlev (1995) at all energies except at the K edge where resonances give an enhancement to the $R$-matrix results. The experimental data point at the $\mathrm{K}$ edge seems to confirm this enhancement, although for the other sequences the Veigele data at the K edge are in good agreement with Verner \& Yakovlev (1995). Except for the area in the immediate vicinity of the K edge, there is similarly good agreement between all three results for the other elements.

Our results are to be used in the XSTAR program (Bautista \& Kallman 2001) for modeling photoionized plasmas. To prepare for this application, we convolve our data with a Gaussian and extrapolate to high energies. As these calculations are focused only on the resonance features near the K edge, the cross section data from the Opacity Project (Seaton et al. 1994) are used for the L-edge region. The two data sets are joined together by hand at an energy between the L and K edges.

The energy-dependent width used for the convolution is $\Delta E / E=10^{-3}$ which is representative of current detectors. The accuracy of $R$-matrix calculations starts to degrade at high energies depending on the number of continuum basis functions included. When this happens, we start to see oscillations in the cross section. Therefore, the calculations are carried out to about 1.5 times the $\mathrm{K}$ edge and then extrapolated to $1100 \mathrm{Ry}$ (or $15 \mathrm{keV}$ ). The extrapolation assumes the asymptotic form $\sigma(E)=A E^{-p}$, where the parameters $A$ and $p$ are determined by a least-squares fit to the last $\sim 100$ points of the $R$-matrix data. This method works well except for some weak partial cross section data where we start to see oscillations. To prevent unphysical extrapolation slopes, we enforce a lower limit of $8 / 3$ on $p$ (Cowan 1981). While the extrapolation is not as accurate for these weak partials, it is not likely to affect modeling. 


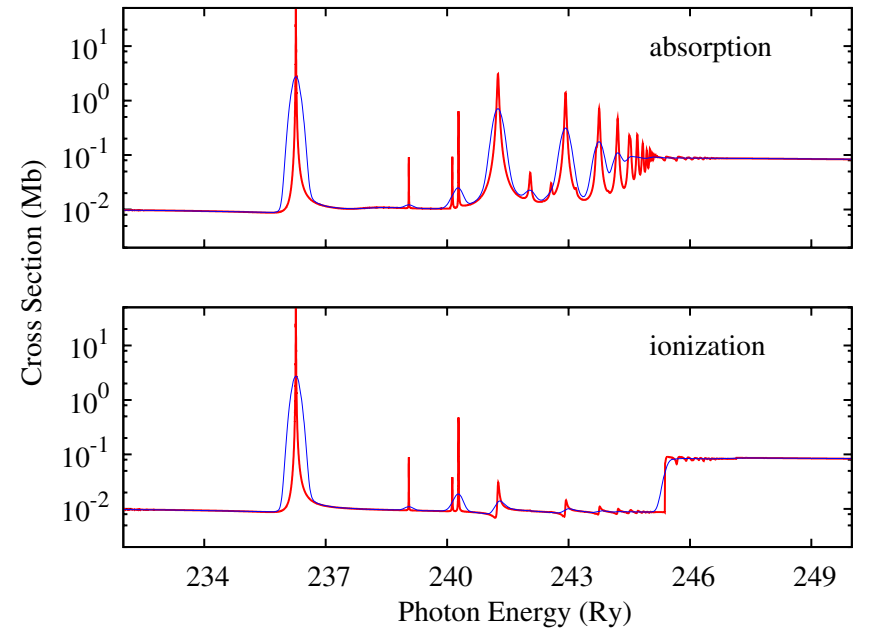

Figure 5. Raw (thick, red) and convolved (thin, blue) cross sections for Mg-like Ar near the $\mathrm{K}$ edge. The top plot shows photoabsorption and the bottom is photoionization. The convolution uses a width of $\Delta E / E=0.001$.

(A color version of this figure is available in the online journal.)

Finally, after convolution and extrapolation, we remove unnecessary data points from each data set. Points that lie on a straight line between the two adjacent points within $1 \%$ are removed. This test is repeated over the entire cross section until no more points are removed. Using this process, we can decrease the number of points in the cross section data from several thousand to a couple hundred, yet still maintain good accuracy with linear interpolation. In Figure 5, we show the raw and convolved total cross sections for Mg-like Ar near the $\mathrm{K}$ edge. The raw data contain over 11,000 points while the convolved data have less than 350 points. The convolved cross sections are not included with this paper, but are available by a request to M.C.W. or XSTAR. The raw cross sections are available as electronic tables attached to this work.

\section{SUMMARY AND CONCLUSIONS}

Total photoabsorption and photoionization cross sections have been computed for the K-shell of all non-neutral ions with 11-17 electrons for $\mathrm{Mg}, \mathrm{Si}, \mathrm{S}, \mathrm{Ar}$, and $\mathrm{Ca}$. Partial photoionization cross sections have also been calculated for the same ions. Radiative and spectator Auger dampings are accounted for in detail and the energy region around the K-threshold was accurately treated for each ion.

We find good agreement in the background total cross section with experiment (Veigele 1973) and the fits of Verner \& Yakovlev (1995). The data provide term-resolved partials which, to our knowledge, have not previously been available. For the Na-like ions, the present results are fully level-resolved.

All data are provided as online tables accompanying this paper and can also be obtained through the XSTAR atomic database (Bautista \& Kallman 2001) ${ }^{5}$ and the Universal Atomic Database. $^{6}$

The data sets provided here together with the energy levels and radiative and Auger rates reported in Palmeri et al. (2008) will help modelers to carry out detailed studies of $\mathrm{K}$ spectra of astrophysically abundant elements.

Support for this research was provided in part by a grant from the NASA Astronomy and Physics Research (APRA) program. P.P. and P.Q. are respectively Research Associate and Senior Research Associate of the Belgian F.R.S.-FNRS. Financial support from this organization is acknowledged.

\section{REFERENCES}

Badnell, N. R. 1986, J. Phys. B: At. Mol. Phys., 19, 3827

Badnell, N. R. 1997, J. Phys. B: At. Mol. Opt. Phys., 30, 1

Bautista, M. A., \& Kallman, T. R. 2001, ApJS, 134, 139

Bautista, M. A., Mendoza, C., Kallman, T. R., \& Palmeri, P. 2003, A\&A, 403, 339

Bautista, M. A., Mendoza, C., Kallman, T. R., \& Palmeri, P. 2004, A\&A, 418, 1171

Berrington, K. A., Burke, P. G., Butler, K., Seaton, M. J., Storey, P. J., Taylor, K. T., \& Yan, Y. 1987, J. Phys. B: At. Mol. Phys., 20, 6379

Berrington, K. A., Burke, P. G., Chang, J. J., Chivers, A. T., Robb, W. D., \& Taylor, K. T. 1974, Comput. Phys. Commun., 8, 149

Berrington, K. A., Burke, P. G., Le Dourneuf, M., Robb, W. D., Taylor, K. T., \& Ky Lan, V. 1978, Comput. Phys. Commun., 14, 367

Berrington, K. A., Eissner, W. B., \& Norrington, P. H. 1995, Comput. Phys. Commun., 92, 290

Boroson, B., Vrtilek, S. D., Kallman, T., \& Corcoran, M. 2003, ApJ, 592, 516

Burke, P. G., Hibbert, A., \& Robb, W. D. 1971, J. Phys. B: At. Mol. Phys., 4, 153

Burke, P. G., \& Seaton, M. J. 1971, Methods in Computational Physics, Vol. 10, Numerical Solution of the Integro-Differential Equations of Electron-Atom Collision Theory (New York: Academic)

Cowan, R. D. 1981, The Theory of Atomic Structure and Spectra (Berkeley, CA: Univ. California Press), 526

García, J., Mendoza, C., Bautista, M. A., Gorczyca, T. W., Kallman, T. R., \& Palmeri, P. 2005, ApJS, 158, 68

García, J., et al. 2009, ApJS, 185, 477

Gorczyca, T. W., \& Badnell, N. R. 1996, J. Phys. B: At. Mol. Opt. Phys., 29, L283

Gorczyca, T. W., \& Badnell, N. R. 2000, J. Phys. B: At. Mol. Opt. Phys., 33, 2511

Hasoglu, M. F., Abdel-Naby, Sh. A., Gorczyca, T. W., Drake, J. J., \& McLaughlin, B. M. 2010, ApJ, 724, 1296

Juett, A. M., \& Chakrabarty, D. 2003, ApJ, 599, 498

Kallman, T. R., Palmeri, P., Bautista, M. A., Mendoza, C., \& Krolik, J. H. 2004, ApJS, 155, 675

Kanter, E. P., Dunford, R. W., Krässig, B., Southworth, S. H., \& Young, L. 2006, Radiat. Phys. Chem., 75, 2174

Kaspi, S., et al. 2002, ApJ, 574, 643

Mendoza, C., Kallman, T. R., Bautista, M. A., \& Palmeri, P. 2004, A\&A, 414, 377

Missavage, D. W., Manson, S. T., \& Daum, G. R. 1977, Phys. Rev. A, 15, 1001

Müller, A., et al. 2009, J. Phys. B: At. Mol. Opt. Phys., 42, 235602

Müller, A., et al. 2010, J. Phys. B: At. Mol. Opt. Phys., 43, 135602

Nasreen, G., Manson, S. T., \& Deshmukh, P. C. 1989, Phys. Rev. A, 40, 6091

Paerels, F. B. S., \& Kahn, S. M. 2003, ARA\&A, 41, 291

Palmeri, P., Mendoza, C., Kallman, T. R., \& Bautista, M. A. 2003a, A\&A, 403, 1175

Palmeri, P., Mendoza, C., Kallman, T. R., Bautista, M. A., \& Meléndez, M. 2003b, A\&A, 410, 359

Palmeri, P., Quinet, P., Mendoza, C., Bautista, M. A., García, J., \& Kallman, T. R. 2008, ApJS, 177, 408

Pradhan, G. B., Jose, J., Deshmukh, P. C., Radojević, V., \& Manson, S. T. 2009, Phys. Rev. A, 80, 053416

Ralchenko, Y., Kramida, A. E., \& Reader, J. NIST ASD Team. 2008, NIST Atomic Spectra Database (version 4.0; Gaithersburg, MD: National Institute of Standards and Technology), http://physics.nist.gov/asd3

Rau, A. R. P. 1976, in Electron and Photon Interactions with Atoms, ed. H. Kleinpoppen \& M. R. C. McDowell (New York: Plenum), 141

Robicheaux, F., Gorczyca, T. W., Pindzola, M. S., \& Badnell, N. R. 1995, Phys. Rev. A, 52, 1319

Scully, S. W. J., et al. 2005, J. Phys. B: At. Mol. Opt. Phys., 38, 1967

Seaton, M. J., Yan, Y., Mihalas, D., \& Pradhan, A. K. 1994, MNRAS, 266, 805

Veigele, W. J. 1973, At. Data, 5, 51

Verner, D. A., \& Yakovlev, D. G. 1995, A\&AS, 109, 125

Witthoeft, M. C., Bautista, M. A., Mendoza, C., Kallman, T. R., Palmeri, P., \& Quinet, P. 2009, ApJS, 182, 127

6 http://heasarc.nasa.gov/uadb 